\title{
Obtaining Minimum Depth Sum of Products from Multiple Constant Multiplication
}

Narges Mohammadi Sarband, Oscar Gustafsson and Mario Garrido

The self-archived postprint version of this journal article is available at Linköping University Institutional Repository (DiVA):

http:/ / urn.kb.se/ resolve?urn=urn:nbn:se:liu:diva- 152653

N.B.: When citing this work, cite the original publication.

Mohammadi Sarband, N., Gustafsson, O., Garrido, M., (2018), Obtaining Minimum Depth Sum of Products from Multiple Constant Multiplication, PROCEEDINGS OF THE 2018 IEEE

INTERNATIONAL WORKSHOP ON SIGNAL PROCESSING SYSTEMS (SIPS), IEEE, , 134-139. https:// doi.org/ 10.1109/ SiPS.2018.8598365

Original publication available at:

https:/ / doi.org/ 10.1109/ SiPS.2018.8598365

Copyright: Institute of Electrical and Electronics Engineers (IEEE)

http:// www.ieee.org/

(C) 2018 IEEE. Personal use of this material is permitted. However, permission to reprint/republish this material for advertising or promotional purposes or for creating new collective works for resale or redistribution to servers or lists, or to reuse any copyrighted component of this work in other works must be obtained from the IEEE. 


\title{
Obtaining Minimum Depth Sum of Products from Multiple Constant Multiplication
}

\author{
Narges Mohammadi Sarband, Oscar Gustafsson, and Mario Garrido \\ Department of Electrical Engineering, Linköping University, SE-581 83 Linköping, Sweden. \\ E-mail: narges.mohammadi.sarband@liu.se, oscar.gustafsson@liu.se, mario.garrido.galvez@liu.se
}

\begin{abstract}
In this work, an approach for transposing solutions to the multiple constant multiplication (MCM) problem to obtain a sum of product (SOP) computation with minimum depth is proposed. The reason for doing this is that solving the SOP problem directly is highly computationally intensive when adder graph algorithms are used. Compared to using sub-expression sharing algorithms, which has a lower computational complexity, directly for the SOP problem, it is shown that the proposed approach, as expected, results in lower complexity for the SOP. It is also shown that there is no obvious way to construct the MCM solution in such a way that the SOP solution has the minimum theoretical depth. However, the proposed approach guarantees minimum depth subject to the MCM solution given as input.

Index Terms-multiple constant multiplication (MCM), shiftand-add, Sum of Product (SOP), minimum depth expansion algorithm, and Vertex reduced SOP adder Graph (VSG).
\end{abstract}

\section{INTRODUCTION}

Multiple constant multiplication (MCM) is the problem to simultaneously multiply one single input data with a set of constant coefficients using adders, subtracters, and shifts [1]. As the implementation cost of adders and subtracters are similar, it is common for brevity to refer to both as adders. A common optimization criteria is to minimize the number of adders, the adder cost, to perform all the multiplications, although it has been shown that the number of cascaded adders, the adder depth, is of importance for the power consumption.

Traditionally, there has been two major classes of algorithms for the MCM problem proposed: adder graph algorithms [2] and sub-expression sharing algorithms. The algorithms based on adder graphs have the benefit of not being dependent on the selection of number representation. Also, these generally consists of two stages: one optimal and one possibly suboptimal part. If only the optimal part is used, the solution is known to be optimal. However, the major challenge, and difference between proposed algorithms, is the sub-optimal part. Sub-expression sharing algorithms on the other hand depends heavily on the chosen representation of the coefficients [3], [4], although several techniques have been introduced to mitigate this issue [5], [6] Also, there are several other sources of suboptimality that require heavy computational efforts to mitigate [7]. However, a major benefit of sub-expression sharing is that the process is rather straightforward and that the time complexity in its basic form is low.
The typical motivating example for MCM is transposed direct form FIR filters. It is well established that such a filter can be transposed to a direct form FIR filter (as it is indeed a transposed version of the direct form FIR filter), and that an MCM solution can be transposed to obtain a sumof-product (SOP) computation. However, how to practically transpose the MCM solution to obtain an SOP computation, taking the depth into account and the properties of such an operation has not been taken into consideration. Although the SOP computation can be solved directly, it should be noted that the computational complexity for adder graph algorithms increases rapidly with an increasing number of inputs. Hence, for SOPs with many inputs, it is extremely time consuming to solve the SOP problem directly using adder graph algorithms, something that can be observed in, e.g., constant matrix-vector multiplication [8]. For sub-expression sharing problems, the number of inputs is not a major contributor to the solutions time. Also, it is possible to take the adder depth into easy consideration. However, the resulting complexity is typically higher compared to what can be obtained solving the MCM problem using an adder graph algorithm.

In this work, we propose a systematic algorithm for obtaining SOP computations from the MCM computation. From some perspective, this is "only" a matter of transposing the MCM solution. However, the proposed approach guarantees that the adder depth is minimal subject to the MCM solution. Furthermore, some properties of the resulting SOP solution are illustrated. Although the proposed approach is here only described for SOPs, it can be extended to matrix-vector multiplication as well, solving for a matrix with fewer columns than rows and transposing the solution using the proposed approach.

In the next section, the algorithm is described in a stepby-step fashion. Special focus is put on how to map the vertex reduced adder graph obtained from transposition to a minimum depth solution with two-input adders. Then, results are presented in Section III, results are presented and the proposed approach is compared to two variants of solving the SOP problem directly using sub-expression sharing.

\section{Proposed Algorithm to Convert MCM to SOP}

In this paper, a systematic algorithm has been used to calculate the sum of products of $N$ inputs by $N$ constant coefficients, using MCM algorithm and transposition theory. The 


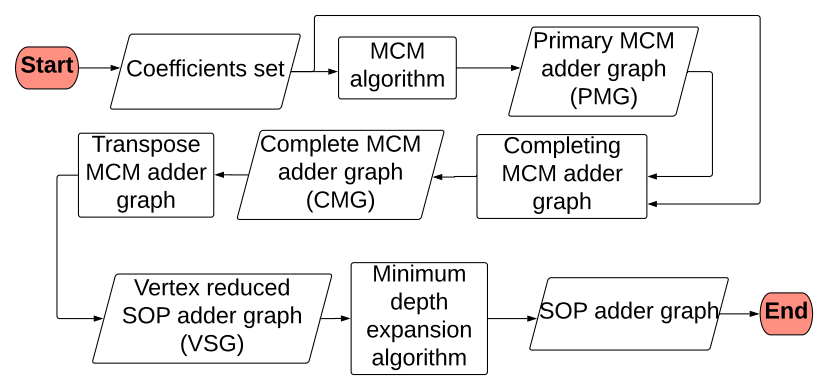

Fig. 1. Proposed steps for producing an SOP by solving the corresponding MCM problem, transposition and minimum depth adder expansion.

flowchart of this process is shown in Fig. 1. This process are explained in following using an example, that $\{7,14,8,-8,4\}$ are a constant coefficients set.

\section{A. Obtaining a Complete MCM Adder Graph}

To produce a SOP adder graph, first, $N$ constant coefficients are given to MCM algorithm which generates a MCM adder graph. A MCM adder graph is a directed graph, in which edges have orientations and it is defined as $(V, E)$. The elements of a set $V$ represent nodes of the graph and $E$ represents edges set. A set $E$, is a set of triple arrows $(a, b, c)$ which means that there is a connection from node $a$ to node $b$ with the weight $c$. In a MCM adder graph, nodes and weights represent adders and shifts, respectively. In this paper, a created MCM adder graph by MCM algorithm is called a primary MCM adder graph (PMG). A PMG is involved the relation of such coefficients that need both shifts and adds and it does not contain coefficients which can be produced, using just shift. A PMG is not also involved negative coefficients, one, zero, and repeated coefficients. In a SOP adder graph, all constant coefficients should be considered, to be multiplied by their corresponding inputs.

Therefore, completing adder graph is the first step which is introduced in this paper. It is used to produce a MCM adder graph which has all coefficients, based on the produced PMG. It produces, negative coefficients, repeated coefficients, coefficients with value one. This step also creates all coefficients as $B$, that are $B=a \times 2^{s}$ in which $a$ is either an input, an internal node, or a output in a PMG. Created PMG and coefficients set are given to completing adder graph step as inputs. It produces complete MCM adder graph (CMG), which has all coefficients. In a CMG, set $V$ is defined as $V=\left\{I, A_{1}, A_{2}, \ldots, A_{k}, O_{1}, O_{2}, \ldots, O_{n}\right\}$, in which node $I$, represents input of MCM adder graph, $A_{i}$ is an internal node in a CMG, and $O_{i}$ is a $i t h$ output of a CMG.

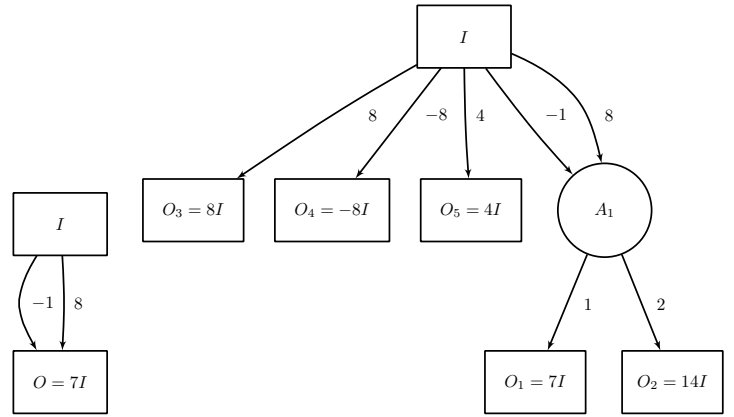

(a)

(b)

Fig. 2. (a) Primary MCM adder graph (PMG) and (b) complete MCM adder graph (CMG) for coefficient set $\{7,14,8,-8,4\}$.

To solve the example, first, the coefficients $\{7,14,8,-8,4\}$ are given to MCM algorithm as an input set and it produces the related PMG which is shown in Fig. 2(a). For the above example, as Fig. 2(a) shows, the MCM algorithm has produced a graph with one output which represents coefficient 7 and other coefficients have been missed. Completing adder graph step for the above example, produces relation of the coefficients $\{7,14,8,-8,4\}$. The CMG, for the above example is shown in Fig. 2(b) which has all coefficients, in comparison with the PMG, shown in Fig. 2(a).

\section{B. Transposing the Complete MCM Adder Graph}

Transposition reverses a signal flow graph of a structure, so the direction of all branches is reversed and inputs with outputs are interchanged. Furthermore, adders and branches are replaced with each other. It keeps same transfer function of the original structure but number of arithmetic operation, as well as numerical properties are different from the original one [9]-[11].

Transposition can be used to generate a sum-of-product (SOP) network, if it is applied to a MCM network [12]. Transposing a single-input- $N$-output MCM network will produce an $N$-input-single-output SOP network. The relation between the number of adders is (1) [12].

$$
\operatorname{Adders}_{\mathrm{SOP}}=\operatorname{Adders}_{\mathrm{MCM}}+N-1 .
$$

The graph resulting from transposition may have more than two incoming vertices to each node, similar to the vertex reduced adder graph in [13]. In addition, for consistency with the complete adder graph format, we change the output node to an adder node and connect a new output node to this node. This graph is here denoted a vertex reduced SOP adder graph (VSG). Transposing the previous example in Fig. 2(b), creates the VSG shown in Fig. 3.

\section{Minimum Depth Expansion}

Converting multiple input adders of a VSG into two-input adders, is the final step of producing a SOP adder graph, which is implemented by minimum depth expansion algorithm. This algorithm changes a $N$-input adder in a VSG to $N-1$ twoinput adders. This converting uses a method that produces the 


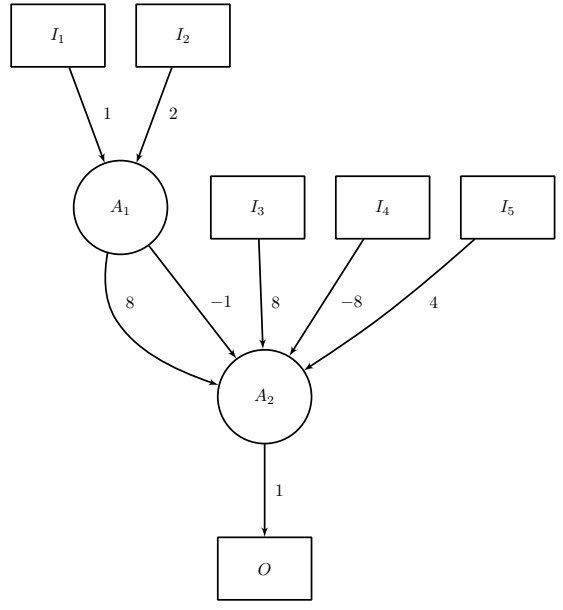

Fig. 3. Vertex reduced SOP adder graph (VSG) created by transposing the complete MCM adder graph in Fig. 2(b), as discussed in Section II-B.

minimum depth for a SOP adder graph. Transposition theory represents, which branches, or inputs of a CMG should be added together to result a SOP adder graph. Therefore, depth of a SOP adder graph is not dependent on transposition theory and it is related to the method which is used in changing, multiple-input adders of a VSG to two-input adders in a SOP adder graph.

To get the minimum depth in addition of $K$ elements, all $K$ elements should be ready from the first step. In a VSG, there are $K$ elements which should be added with each other, but their availability depth is different. These are just, inputs edges, that are available from the beginning, $($ depth $=0)$. But there are branches that, they would be ready after some calculations. To achieve the minimum depth for addition of some edges together with different depth, the minimum depth expansion algorithm is offered which is described in the Alg. 1.

This algorithm guarantees that, it would create a SOP adder graph for the related VSG with the minimum depth. To get the minimum depth, this algorithm uses a method which manages the availability depth of the all nodes. Furthermore, the algorithm uses ASAP 'As-Soon-As-Possible' addition algorithm, in which the addition would be done whenever both edges connected to a adder are ready.

This algorithm is applied to the example and creates the minimum depth for the SOP adder graph based on the received VSG in Fig. 3. The SOP adder graph is shown in Fig. 4.

\section{RESULTS}

To solve the MCM problem, the algorithm in [2] is used. As all MCM algorithms aiming at the lowest adder cost, it can sometimes lead to a higher adder depth. However, the only way to reduce the adder depth is in most cases to increase the adder cost. While it could be interesting to further look into this trade-off and how it affects transposition, it is not part of the current work. However, in the next section some properties of the relation between the depth of the MCM solution and the resulting SOP solution are illustrated. Then, the approach

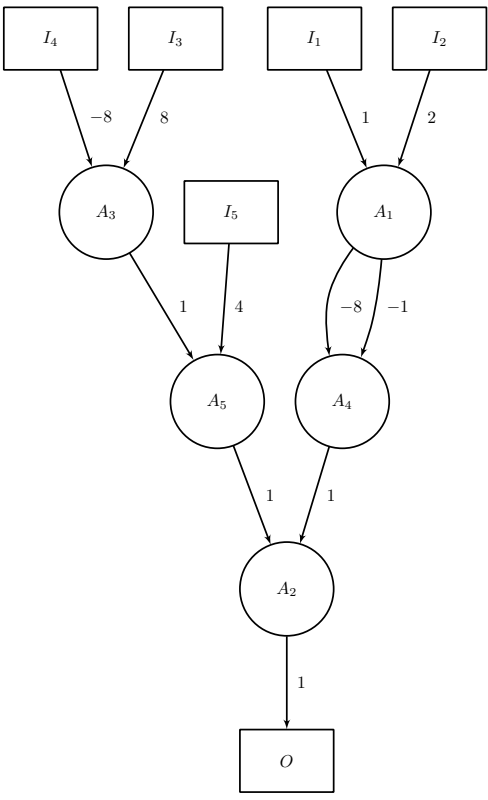

Fig. 4. The resulting SOP adder graph for the coefficient set $\{7,14,8,-8,4\}$ after applying the minimum depth expansion algorithm in Section II-C to the graph in Fig. 3.

solving the MCM problem and using the proposed approach to obtain the SOP solution is compared to solving the SOP problem directly using sub-expression sharing.

\section{A. Adder Depth in MCM and SOP}

To see the relation between the adder depths for the MCM and SOP solutions, 1000 random coefficient sets were considered for 20 or 50 coefficients and 12 or 16 bits coefficients. The results are shown in Fig. 5. First, it can be observed that the depth of the SOP solution is never smaller than the depth of the MCM solution. This is not surprising considering the underlying graph structure: the number of intermediate nodes between the input and one output will never decrease by transposing the graph. It is also possible to see that the adder depth of the MCM solution does not have that big impact on the adder depth of the SOP solution. For example, considering Fig. 5(a) the adder depth of most MCM solutions are between 2 and 14, while the adder depth of the SOP solution is between 8 and 15 , more or less without any obvious relation (except for the bound observed initially. Hence, an MCM solution with adder depth 2 may give an SOP solution with adder depth 13, just as an MCM solution with an adder depth of 9 may give an SOP solution with an adder depth of 10 .

To further illustrate this, consider the SOP computation of the set $\{3,7,21\}$. Its MCM form can be optimally realized using an adder cost 3 and adder depth 2 as shown in Fig. 6(a). The SOP form shown in Fig. 6(b) results in an adder depth 4 and, as expected from (1), an adder cost 5 . Introducing an additional adder, resulting in the MCM form shown in Fig. 6(c) still results in adder depth 2 . However, when transposing this graph to obtain the SOP form, a solution with adder depth 3 is obtained (and expected adder cost 6). This further establishes 


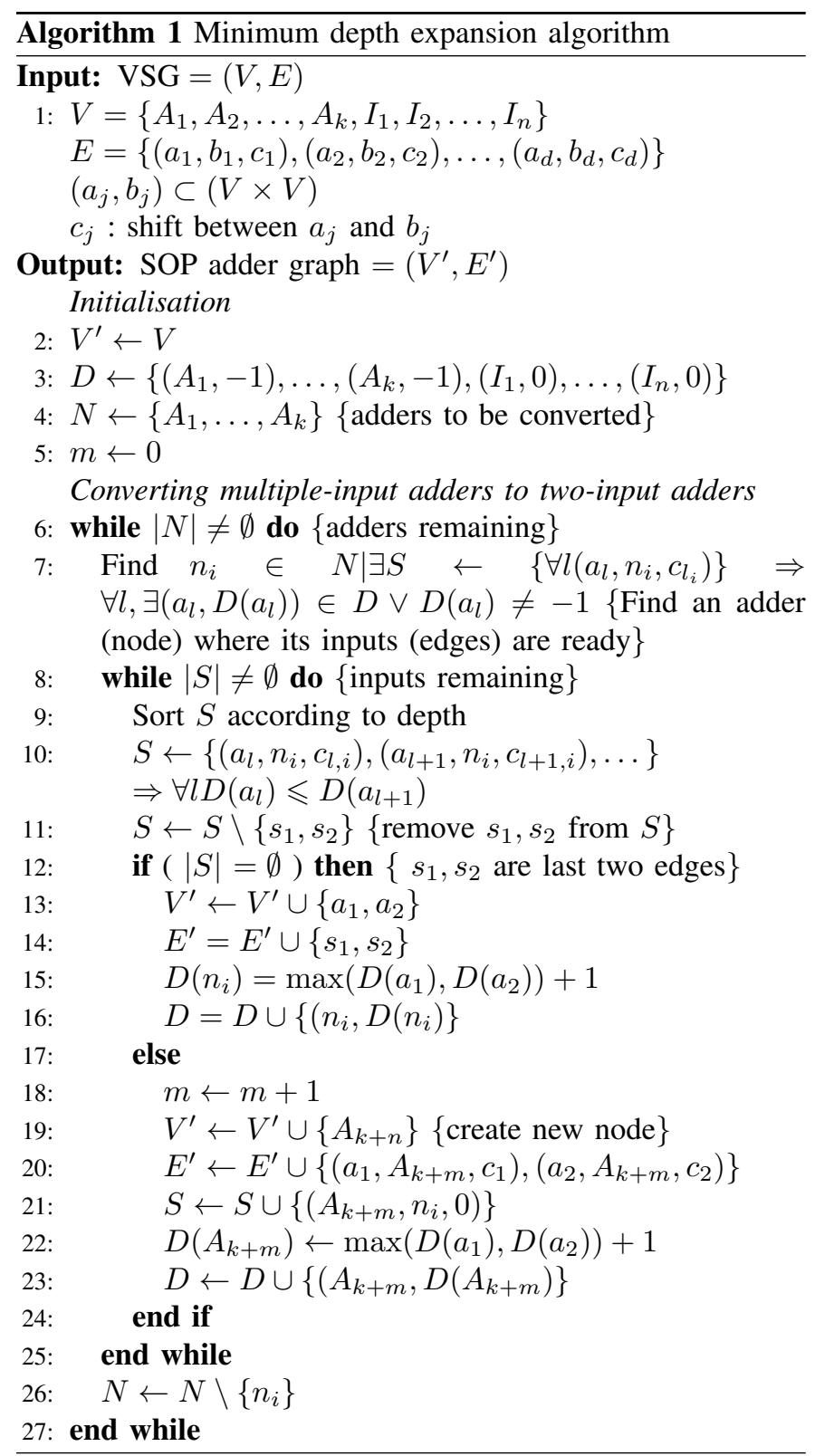

that it is not (yet) clear what the preferred properties of the MCM solution is to obtain a low depth SOP solution.

\section{B. Comparison to Sub-Expression Sharing}

For comparison, an implementation of common two-term subexpression sharing is used. The algorithm is similar to that in [14] and uses CSD representation of the coefficients. However, two different strategies of selecting the subexpression to share was used [15]. The first one, denoted SES in the following, picks the most common subexpression, as most algorithms do, with the purpose to minimize the adder cost and is therefore close to [14]. The second, denoted SES2, gives priority to minimum adder depth in the selection, therefore guaranteeing a minimum adder depth for the complete solution, while still sharing sub-expressions.

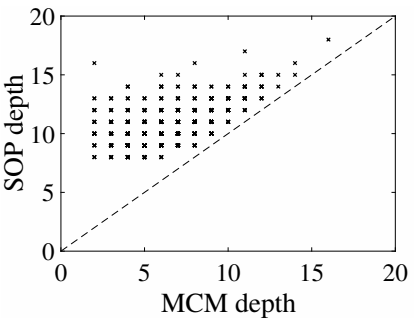

(a)

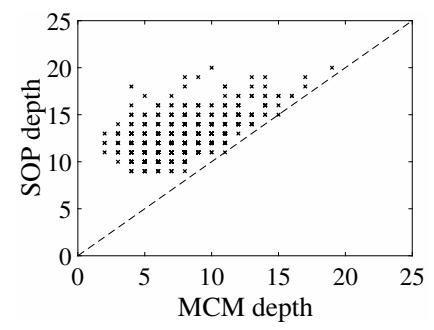

(c)

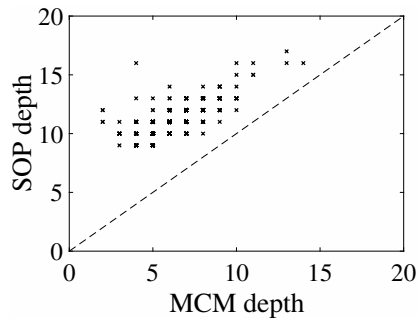

(b)

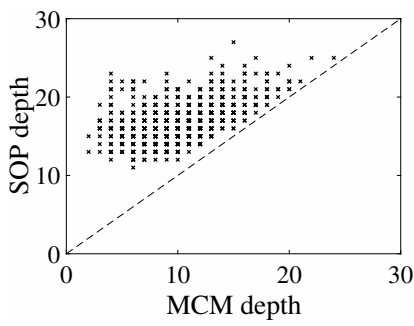

(d)
Fig. 5. Relation between the depth of an MCM solution and the transposed SOP solution for: (a) $W=12, N=20$, (b) $W=12, N=50$, (c) $W=16$, $N=20$, and (d) $W=16, N=50$

In Fig. 7, the average adder costs for the different word lengths and different number of coefficients are shown. As expected, the SOP solution provides the lowest number of adders as the underlying MCM algorithm is based on adder graphs and therefore not representation dependent. As can be seen, the benefit of the proposed approach increases with the word length. It is also clear that the SES approach results in fewer adders compared to the minimum depth SES2 approach. The additional depth constraint comes at a price.

The resulting average adder depths are shown in Fig. 8. First, the step-like behavior of the minimum depth SES2 algorithm is not surprising. The minimum adder depth of an SOP is

$$
\left\lceil\log _{2}\left(\sum_{n=1}^{N} C\left(X_{n}\right)-1\right)\right\rceil,
$$

where $C\left(X_{n}\right)$ is the number of non-zero digits of coefficient $X_{n}$ [1]. Hence, with random coefficients, it is expected that the average value will follow the expectation values of the equation closely. When comparing the methods to obtain the SOPs, it is seen that the average adder depth of the proposed method will go below that of SES given enough coefficients. It also indicates that the average adder depth of SOP settles at a limit determined by the number of coefficient bits, given that it does not violate the lower bound. It should be noted that the use of a different MCM algorithm may change this behavior.

As earlier discussed, not only the adder cost, but also the adder depth contributes to the power consumption. Hence, the power consumption is a combination of Figs. 7 and 8 . Although a more careful analysis is required to be certain, it is at least possible to state that for many cases the proposed approach is likely to result in a lower power consumption 


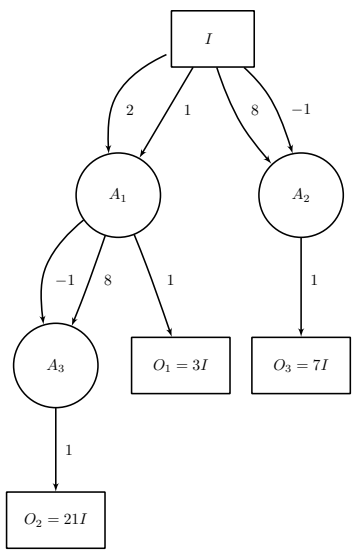

(a)

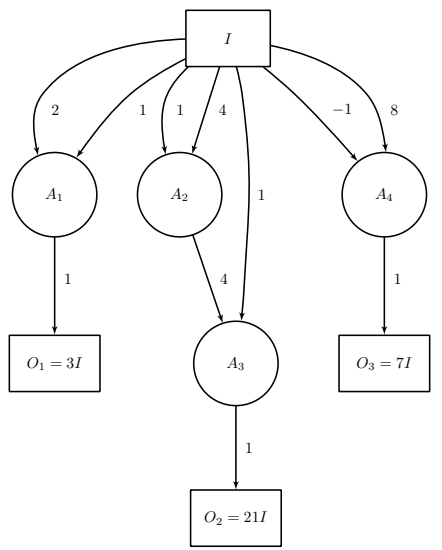

(c) (a)

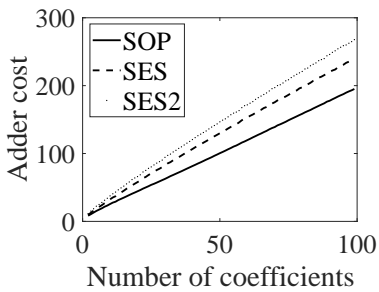

(c)

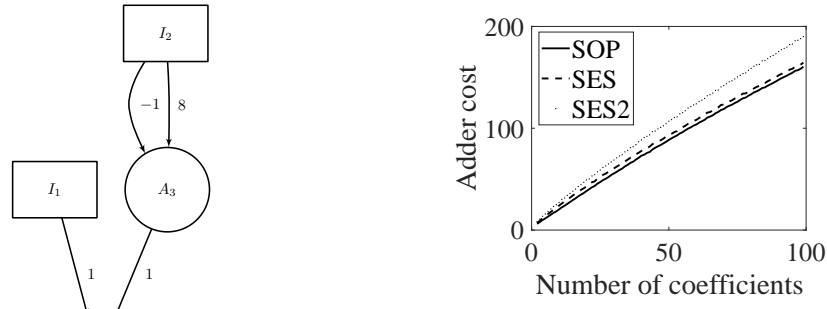

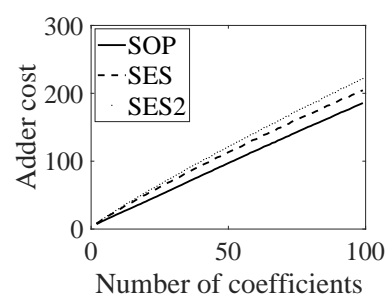

(b)

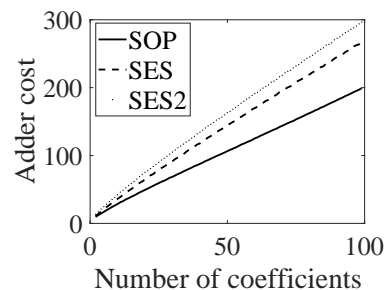

(d)
Fig. 7. Average adder cost for SOP, SES, and SES2 with different word lengths: (a) $W=8$, (b) $W=10$, (c) $W=12$, and (d) $W=14$.

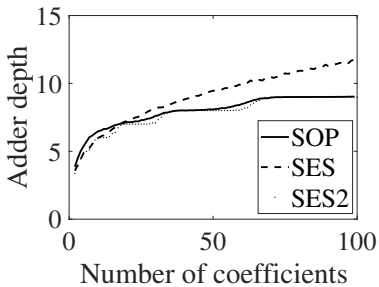

(a)

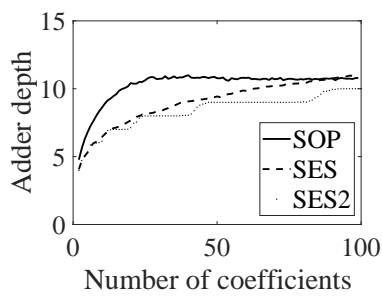

(c)

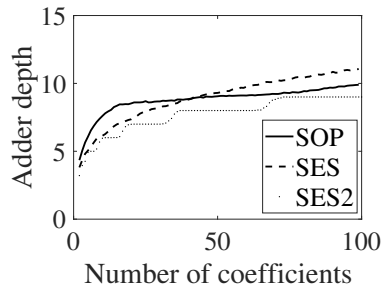

(b)

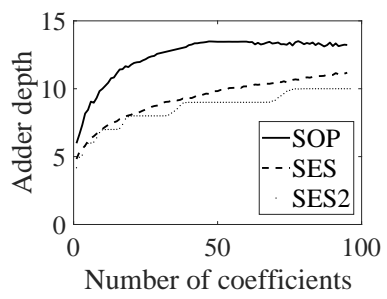

(d)
Fig. 8. Average adder depth for SOP, SES, and SES2 with different word lengths: (a) $W=8$, (b) $W=10$, (c) $W=12$, and (d) $W=14$.

Fig. 6. Realization of the coefficient set $\{3,7,21\}$. (a) Optimal MCM solution, (b) SOP solution from (a) with adder depth 4, (c) MCM solution with extra adder, and (d) SOP solution from (c) with adder depth 3 .

than the subexpression sharing based solutions. Especially this should hold for many coefficients, where the savings in adder cost is large and the increase in adder cost is low. However, it should be noted that the results are average values. Hence, one may expect individual cases where this relation is even more clear, and, naturally, also the opposite.

\section{CONCLUSION}

In this work a method to systematically obtain a sum of products (SOP) solution from a multiple constant multipli- cation (MCM) solution has been proposed. While it is well known that it is possible to perform this transformation, the proposed approach guarantees minimum adder depth subject the MCM solution. This finds application in many different scenarios where a sum of product with constant coefficients must be computed in parallel. For example, in digital filters, transforms, and convolutional neural networks.

The benefit of the approach is that it is much faster compared to solving the SOP directly using adder graph algorithms, while the adder cost is lower compared to the computationally more efficient algorithms based on subexpression sharing. The approach can be modified to be applied to constant matrix-vector computations, although it is beyond the 
scope of this work to consider that in detail.

The results show that the adder depth of the resulting SOP solution does not significantly depend on the depth of the MCM solution, although it provides a strict lower bound. Furthermore, compared to subexpression sharing methods, the proposed approach provides significantly fewer adders, although at a higher adder depth on average. However, both the factors depends on the MCM algorithm used and a future problem is to look into which properties of the MCM solutions are that results in a low adder depth for the SOP solution.

The proposed method provides an efficient way to realize low complexity SOP computations without solving the much more computationally intensive SOP problem directly. The resulting SOP computation is minimum depth subject to the MCM solution provided.

\section{REFERENCES}

[1] O. Gustafsson, "Lower bounds for constant multiplication problems," IEEE Trans. Circuits Syst. II, vol. 54, no. 11, pp. 974-978, Nov. 2007.

[2] — , "A difference based adder graph heuristic for multiple constant multiplication problems," in Proc. IEEE Int. Symp. Circuits Syst. IEEE, 2007, pp. 1097-1100.

[3] A. G. Dempster and M. D. Macleod, "Using all signed-digit representations to design single integer multipliers using subexpression elimination," in Proc. IEEE Int. Symp. Circuits Syst., vol. 3. IEEE, 2004, pp. 165-168.

[4] M. Imran, K. Khursheed, M. O'Nils, and O. Gustafsson, "On the number representation in sub-expression sharing," in Proc. Int. Conf. Signals Electron. Syst. IEEE, 2010, pp. 17-20.

[5] J. Thong and N. Nicolici, "Time-efficient single constant multiplication based on overlapping digit patterns," IEEE Trans. VLSI Syst., vol. 17, no. 9 , pp. $1353-1357$, Sep. 2009.

[6] O. Gustafsson, K. Khursheed, M. Imran, and L. Wanhammar, "Generalized overlapping digit patterns for multi-dimensional sub-expression sharing," in Proc. IEEE Int. Conf. Green Circuits Syst. IEEE, 2010, pp. $65-68$

[7] L. Aksoy, E. Da Costa, P. Flores, and J. Monteiro, "Exact and approximate algorithms for the optimization of area and delay in multiple constant multiplications," IEEE Trans. Comput.-Aided Design Integr. Circuits Syst., vol. 27, no. 6, pp. 1013-1026, Jun. 2008.

[8] M. Kumm, M. Hardieck, and P. Zipf, "Optimization of constant matrix multiplication with low power and high throughput," IEEE Trans. Comput., vol. 66, no. 12, pp. 2072-2080, Dec. 2017.

[9] J. L. Bordewijk, "Inter-reciprocity applied to electrical networks," Appl. Scientific Research, vol. 6, no. 1, pp. 1-74, Jan. 1957.

[10] B. Bhattacharyya and M. Swamy, "Network transposition and its application in synthesis," IEEE Trans. Circuit Theory, vol. 18, no. 3, pp. 394-397, May 1971.

[11] H. Schmid, "Circuit transposition using signal-flow graphs," in Proc. IEEE Int. Symp. Circuits Syst., vol. 2, 2002, pp. 25-28.

[12] O. Gustafsson and A. G. Dempster, "On the use of multiple constant multiplication in polyphase FIR filters and filter banks," in Proc. IEEE Nordic Signal Process. Symp., Jun. 2004, pp. 53-56.

[13] O. Gustafsson, A. G. Dempster, K. Johansson, M. D. Macleod, and L. Wanhammar, "Simplified design of constant coefficient multipliers," Circuits Syst. Signal Process., vol. 25, no. 2, pp. 225-251, Apr. 2006.

[14] M. Macleod and A. Dempster, "Common subexpression elimination algorithm for low-cost multiplierless implementation of matrix multipliers," Electron. Lett., vol. 40, no. 11, pp. 651-652, 2004.

[15] O. Gustafsson, "Unified and generalized subexpression sharing in arbitrary dimensions," 2018, in preparation. 